\title{
Mitochondria Swelling - a Response to the Use of ACE Inhibitor as an Effect of Cardiomyocytes Stimulation
}

\author{
W. Matysiak, B. Jodłowska-Jędrych \\ Department Histology and Embryology, Medical University of Lublin \\ e-mail: w.matysiak@wp.pl, 11 Street Radziwiłłowska, 20-080 Lublin, Poland
}

Until recently angiotensin converting enzyme (ACE) inhibitors were mainly used in patients treated for arterial hypertension. Nowadays therapeutic indications for drugs of that group have been considerably broadened to include other diseases and pathological conditions: circulatory insufficiency, cardiac dysrrhythmia, myocardial infarction, diabetic micro- and macroangiopathies, nephropathies and central nervous system diseases [1-2].

Studies on other therapeutic applications of converting enzyme inhibitors are still in progress. When administered in the cases of hypertension they not only lower blood pressure but also cause regression of left-ventricular hypertrophy and changes in local vascular resistance, which leads to changes in arteries susceptibility with simultaneous functional and structural changes of arterial system. This may be of importance in coronary heart disease as a result of inhibition of endothelium proliferation. The role of converting enzyme inhibitors in angina pectoris is also being investigated.

Captopril is an angiotensin converting enzyme inhibitor. It blocks the conversion of angiotensin I into angiotensin II, inhibiting renin-angiotensin-aldosterone system. The drug dilates peripheral vessels, reduces peripheral resistance and increases renal blood flow.

The studies of Captopril effects in patients with chronic cardiac insufficiency show increase in minute volume and reduction of peripheral and pulmonary vascular resistance. There also occurs alleviation of symptoms and decrease in mortality.

Captopril (BIOFARM, Poland) was administered to white rats, males (Wistar) in the period of 4 weeks, in the dose of $25 \mathrm{mg} / 24$ hours (half of the therapeutic dose) and in the dose of $75 \mathrm{mg} / 24$ hours (half of the maximum dose). After decapitation sample material was collected from the animals - sections of left and right ventricles of the heart. The sections were evaluated using a transmission electron microscope.

The study results indicate that Captopril administration causes changes in mitochondria structure which are the response to the stimulation of the myocardium cells. A relevant feature observed in the sections collected both from left and right ventricles of the heart from the animals of the experimental group receiving half of the drug maximum dose, was mitochondria swelling (Fig. 1), which may suggest adaptation of those organelle to elevated energy requirement of the cells. The process of cardiomyocytes stimulation leads to the generation of inositol trisphosphate (IP3), which activates calcium canals in sarcoplasmic reticulum, sensitive to IP3. Increase in the concentration of calcium ions stimulates mitochondria response [3-4].

There were observed more numerous glycogen granules agglomerations (Fig. 2), compared to the control group preparations, which made cardiomyocytes more resistant to hypoxia.

Reference materials data imply that ACE inhibitors effectively influence the stabilization of vascular endothelium and the reduction - in pathological states - of the mass of hypertrophied left ventricle of the heart and cause the improvement of myocardium activities. There was proved a positive effect of Captopril on the normalization of the endothelium and stimulation of angiogenesis in rabbits in a hypoxia model [5-6]. 
The conducted experiments imply that the administration of ACE inhibitor (Captopril) in therapeutic doses has a positive effect on the functional state of the myocardium, which is associated with improvement in the conditions of the myocardium work.

References

[1] J. Rezler, Kardiol. Pol. 46 (1997) 455.

[2] P. Gurer et al., Hum. Exp. Toxicol. 18 (1999) 27.

[3] A. Schattener et al., Am. Med. J. Sci. 332 (2001) 236.

[4] M.A El-Missiry et al., J. Appl. Toxicol. 21 (2001) 469.

[5] Yoshiji H. et al., Tumor Biol. 23 (2002) 348.

[6] N.H. Andersen and C.E. Mogensen, Medycyna po Dyplomie. 12 (2003) 95.

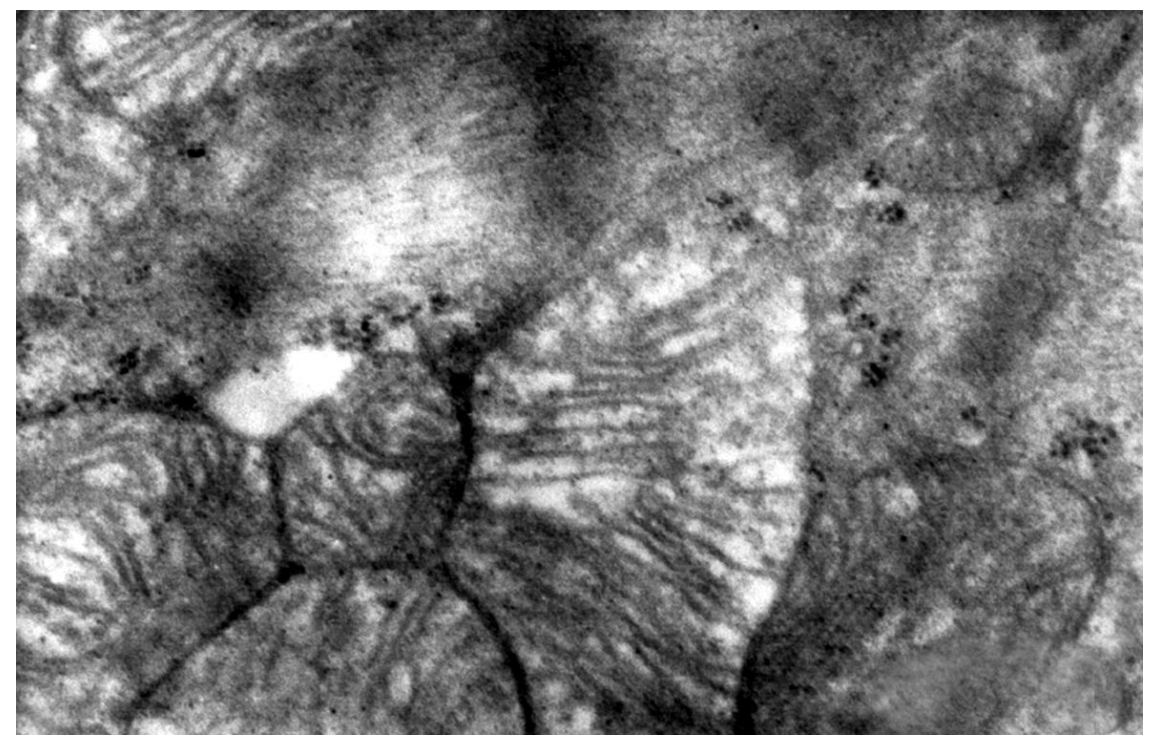

Fig. 1. Mitochondria swelling features in cardiomyocytes. Magn. 14000 x

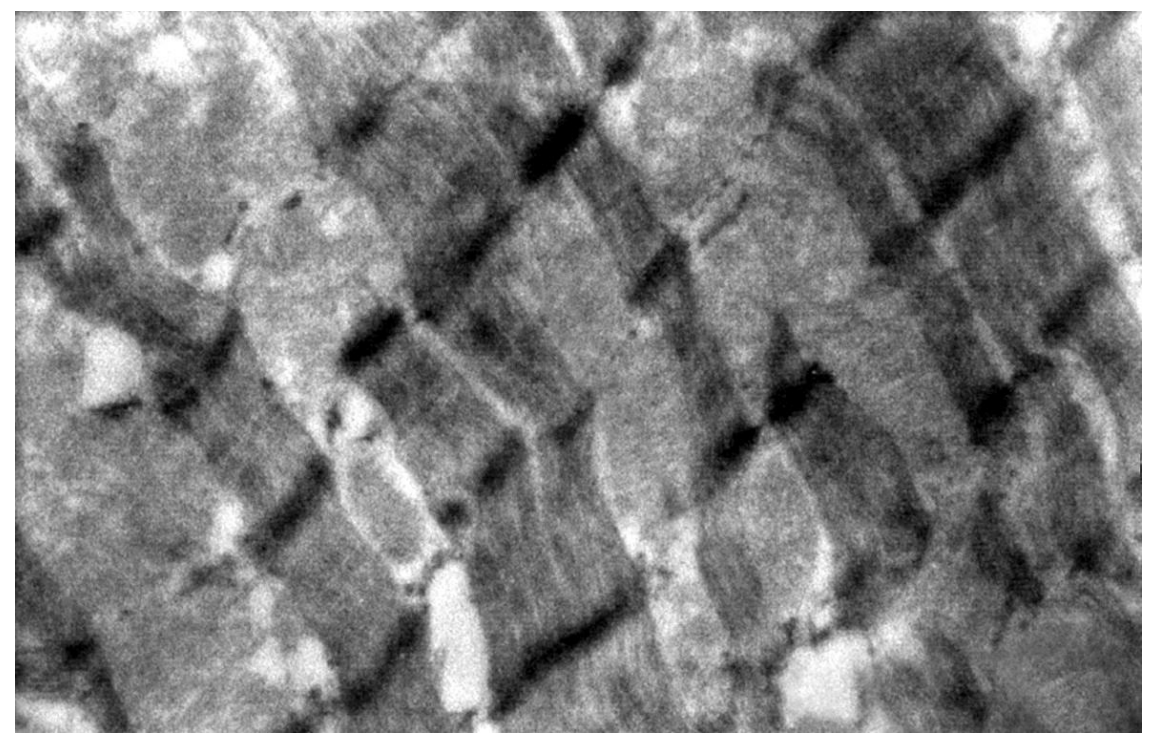

Fig. 2. Glycogen granules present in cardiomyocytes. Magn. 10000x. 